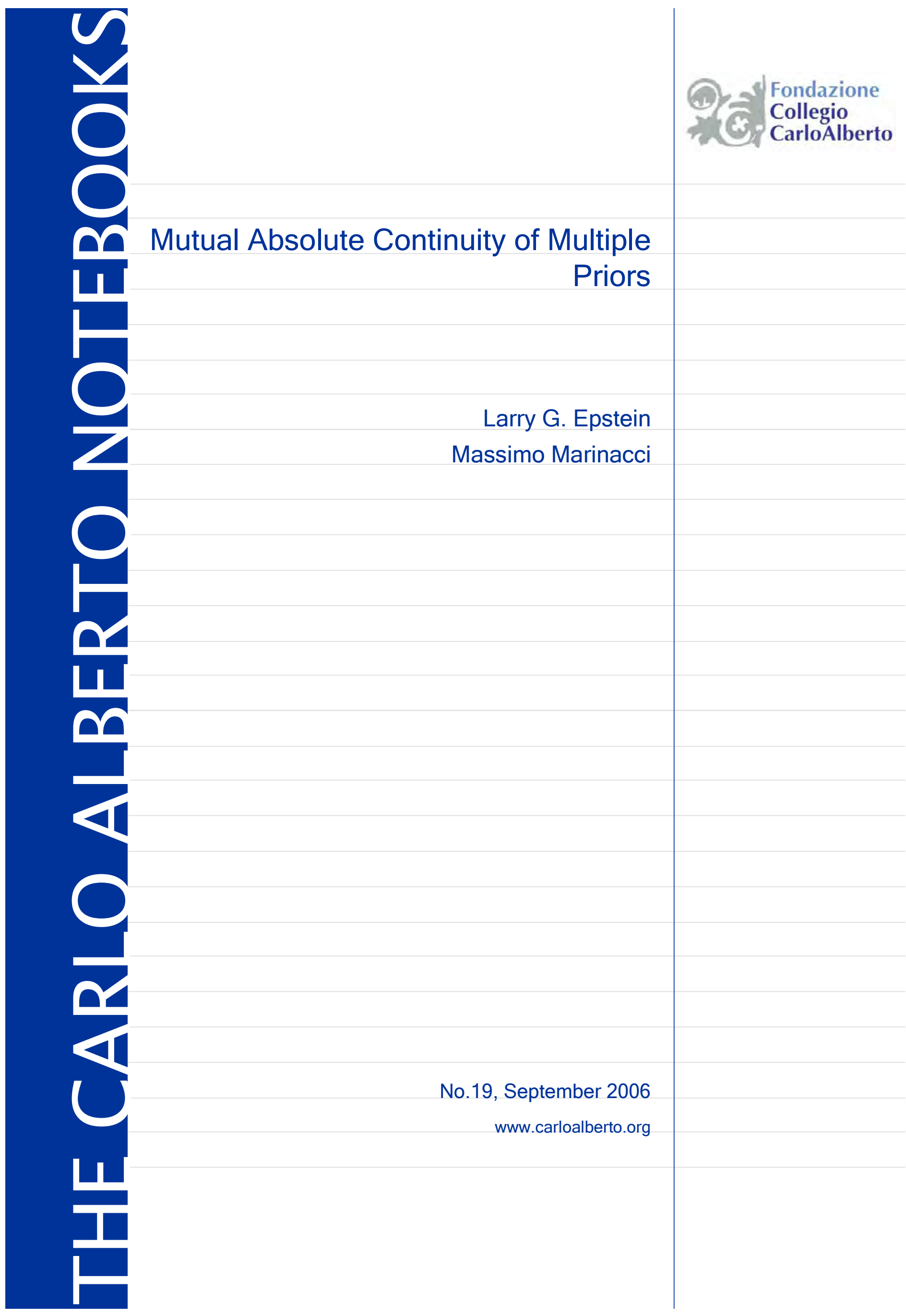




\title{
Mutual Absolute Continuity of Multiple Priors ${ }^{1}$
}

\author{
Larry G. Epstein Massimo Marinacci
}

August 2006

${ }^{1}$ Epstein is at Department of Economics, University of Rochester, USA, lepn@troi.cc.rochester.edu; Marinacci is at Dipartimento di Statistica e Matematica Applicata and Collegio Carlo Alberto, Università di Torino, Italy, massimo.marinacci@unito.it. Epstein gratefully acknowledges the financial support of the NSF (award SES-0611456) and Marinacci that of the Ministero dell'Istruzione, dell'Università e della Ricerca.

(C) 2006 by Larry Epstein and Massimo Marinacci. Any opinions expressed here are those of the authors and not those of the Fondazione Collegio Carlo Alberto. 


\begin{abstract}
This note provides a behavioral characterization of mutually absolutely continuous multiple priors.

JEL classification: D81
\end{abstract}

Keywords: Mutual absolute continuity, Multiple priors 


\section{Introduction}

Beginning with Epstein and Schneider [2], in recent years there has been a growing interest in dynamic versions of the multiple-priors (MP) model of Gilboa and Schmeidler [4], motivated by the importance of dynamic considerations in many economic applications. In these dynamic versions of the MP model a very convenient property that is often assumed is mutual absolute continuity of the priors, that is, their mutual agreement on which events are null. This agreement makes possible dynamically consistent Bayesian updating of the priors, without having to deal with updating on events that only some priors regard as null, something that greatly simplifies the analysis of these dynamic MP models. ${ }^{1}$

Here we provide a behavioral characterization of this agreement property of the priors. Interestingly, the relevant behavioral condition turns out to be the translation into the Savage framework of a condition introduced by Kreps [5] in his seminal paper on menu choices.

\section{Setting}

We use a standard Savage-style setting. Throughout, $\Sigma$ is an event algebra in a state space $S$. Subsets of $S$ are understood to be in $\Sigma$ even where not stated explicitly.

We denote by $\Delta$ the set of all priors, that is, the set of all finitely additive probability measures $P$ on $\Sigma$. Two such measures $P_{1}$ and $P_{2}$ are mutually absolutely continuous when, for all $E \in \Sigma, P_{1}(E)=0$ if and only if $P_{2}(E)=0$.

We denote by $X$ the space of consequences. An act is a map $f: S \rightarrow X$ and it is simple when it is finite valued; $L_{0}$ denotes the set of all simple $\Sigma$-measurable acts.

The decision maker has a preference relation $\succsim$ on $L_{0}$, which in turn induces a preference over $X$, obtained in the standard way by identifying consequences with constant acts.

Say that an event $E$ is null (resp. universal) if $x E y \sim y$ (resp. $x E y \sim x$ ) for all $x, y \in X$ such that $x \succ y$. Here $x E y$ is the act that pays $x$ if $E$ obtains, and $y$ otherwise.

A binary relation $\succsim$ on $L_{0}$ is a multiple-priors $(\mathrm{MP})$ preference relation if there exists a utility index $u: X \rightarrow \mathbb{R}$, and a non-empty weak*-compact set $C \subseteq \Delta$ such that $\succsim$ is represented by the preference functional $V: L_{0} \rightarrow \mathbb{R}$ defined by

$$
V(f)=\min _{P \in C} \int u(f(s)) d P(s), \quad \forall f \in L_{0} .
$$

\footnotetext{
${ }^{1}$ Similar considerations apply to the closely related topic of dynamic coherent risk measures (see $[1])$.
} 
Subjective expected utility (SEU) preferences are the special case of singleton sets of priors $C$.

For MP preferences, an event $E$ is null iff $P(E)=0$ for some $P \in C$, while $E$ is universal iff $P(E)=1$ for all $P \in C$. Hence, the complement of a universal event is null, but the converse is in general false.

\section{The Axiom and Results}

Given any two consequences $x$ and $y$, let

$$
x \vee y= \begin{cases}x & \text { if } x \succsim y \\ y & \text { else }\end{cases}
$$

and given any two acts $f$ and $g$, define the act $f \vee g$ by

$$
(f \vee g)(s)=f(s) \vee g(s), \quad \forall s \in S
$$

The next axiom translates the Kreps axiom (see [5, Eq. (1.5)]) into our setting.

Axiom 1 (General Kreps (GK)) For all $f, f^{\prime}, g \in L_{0}$,

$$
f \sim f \vee f^{\prime} \Longrightarrow f \vee g \sim(f \vee g) \vee f^{\prime}
$$

In every state, the act $f \vee f^{\prime}$ gives the better of the two outcomes associated with $f$ and $f^{\prime}$. Thus say that $f \vee f^{\prime}$ weakly improves $f$ in 'the direction' $f^{\prime}$. GK requires that if an improvement of $f$ in direction $f^{\prime}$ has no value, then the same must be true for an improvement in direction $f^{\prime}$ of any act (here $f \vee g$ ) that improves $f$. The scope of this seemingly innocuous axiom will be fully understood in light of the results to follow.

First observe that SEU preferences always satisfy the GK axiom: $f \sim f \vee f^{\prime} \Longrightarrow$

$$
\begin{gathered}
\int u(f) d P=\int u\left(f \vee f^{\prime}\right) d P=\int\left(u(f) \vee u\left(f^{\prime}\right)\right) d P \Longrightarrow \\
u\left(f^{\prime}(s)\right) \leq u(f(s)) P \text {-a.e. } \Longrightarrow \\
u\left(f^{\prime}(s)\right) \vee u(g(s)) \leq u(f(s)) \vee u(g(s)) \text { P-a.e. } \Longrightarrow \\
f \vee g \sim(f \vee g) \vee f^{\prime} .
\end{gathered}
$$

In general MP preferences do not satisfy GK, as the next example shows. 
Example 1 Let $S=\{1,2\}$ and $X \subseteq \mathbb{R}$. Consider acts $f=\left(x_{1}, x_{2}\right), f^{\prime}=\left(x_{1}^{\prime}, x_{2}^{\prime}\right)$ and $g=\left(y_{1}, y_{2}\right)$, where

$$
x_{1}^{\prime}<y_{2}<x_{1}<x_{2}<x_{2}^{\prime}<y_{1} .
$$

We have $f \vee f^{\prime}=\left(x_{1}, x_{2}^{\prime}\right), f \vee g=\left(y_{1}, x_{2}\right)$, and $(f \vee g) \vee f^{\prime}=\left(y_{1}, x_{2}^{\prime}\right)$.

Consider the case of complete ignorance and risk neutrality: $u(x)=x$ and $C=$ $\{(p, 1-p): p \in[0,1]\}$. Then, contrary to GK,

$$
V(f)=V\left(f \vee f^{\prime}\right)=x_{1} \quad \text { and } \quad V(f \vee g)=x_{2}<x_{2}^{\prime}=V\left(f \vee g \vee f^{\prime}\right)
$$

The following result characterizes MP preferences that satisfy GK, and it shows that the latter is the sought-after behavioral characterization of the mutual absolute continuity of priors.

Theorem 1 For any MP preference $\succsim$, the following properties are equivalent:

(i) $\succsim$ satisfies $G K$;

(ii) all measures in $C$ are mutually absolutely continuous;

(iii) any event $E$ is null iff its complement $E^{c}$ is universal.

Proof. The equivalence of (ii) and (iii) is easily established. We prove that (i) and (ii) are equivalent.

(ii) implies (i): Assume that all measures in $C$ are mutually absolutely continuous. Suppose that $f \sim f \vee f^{\prime}$. Then there exists $P^{*} \in C$ such that

$$
\begin{aligned}
\min _{P \in C} \int u\left(f \vee f^{\prime}\right) d P & =\min _{P \in C} \int\left(u(f) \vee u\left(f^{\prime}\right)\right) d P=\int\left(u(f) \vee u\left(f^{\prime}\right)\right) d P^{*} \\
& \geq \int u(f) d P^{*} \geq \min _{P \in C} \int u(f) d P=\min _{P \in C} \int u\left(f \vee f^{\prime}\right) d P .
\end{aligned}
$$

Hence,

$$
\begin{gathered}
\int u(f) d P^{*}=\int\left(u(f) \vee u\left(f^{\prime}\right)\right) d P^{*} \Longrightarrow \\
u\left(f^{\prime}(s)\right) \leq u(f(s)) P^{*} \text {-a.e. } \Longrightarrow \\
u\left(f^{\prime}(s)\right) \vee u(g(s)) \leq u(f(s)) \vee u(g(s)) P^{*} \text {-a.e. } \Longrightarrow \\
u\left(f^{\prime}(s)\right) \vee u(g(s)) \leq u(f(s)) \vee u(g(s)) P^{*} \text {-a.e. } \forall P \in C \Longrightarrow \\
u\left(f^{\prime}(s)\right) \vee u(g(s)) \vee u(f(s))=u(f(s)) \vee u(g(s)) P^{*} \text {-a.e. } \forall P \in C \Longrightarrow \\
f \vee g \sim(f \vee g) \vee f^{\prime} .
\end{gathered}
$$

(i) implies (ii): Assume that $\succsim$ satisfies GK but that not all measures in $C$ are mutually absolutely continuous. Then there exist an event $E$ and $P_{1}, P_{2} \in C$ such that 
$P_{1}(E)=0<P_{2}(E)$. Let $\bar{P} \in C$ be such that $\bar{P}\left(E^{c}\right) \leq P\left(E^{c}\right)$ for all $P \in C$. Then $\bar{P}(E) \geq P_{2}(E)>0$, and so $\bar{P}\left(E^{c}\right)<1$.

Wlog let $x$ and $y$ be outcomes such that $u(x)=1$ and $u(y)=0$. Since $(x E y) \vee y=$ $x E y$, we have $V(x E y \vee y)=V(x E y)=P_{1}(E)=0$. Hence, $x E y \vee y \sim y$. On the other hand, $x E y \vee y \vee x E^{c} y=x$ and $y \vee x E^{c} y=x E^{c} y$, so that

$$
V\left(x E y \vee y \vee x E^{c} y\right)=1>\bar{P}\left(E^{c}\right)=V\left(y \vee x E^{c} y\right),
$$

and $x E y \vee y \vee x E^{c} y \succ y \vee x E^{c} y$, contradicting GK.

Remark 1 Partial versions of Theorem 1 holds for more general classes of preferences. For example, (i) implies (iii) for the biseparable preferences of [3], while (ii) implies (i) for the variational preferences of [6] (in this case condition (ii) becomes the mutual absolute continuity of the probabilities belonging to the effective domain of the function $c$ used in their representation).

It is natural to understand GK as follows: suppose that $f \sim f \vee f^{\prime}$. Then it must be that the set of states where $f^{\prime}(s)$ is strictly preferred to $f(s)$ is "impossible," and therefore that the event $\left\{s: f(s) \succsim f^{\prime}(s)\right\}$ is "certain." Then the larger event $\left\{s: f(s) \vee g(s) \succsim f^{\prime}(s) \vee g(s)\right\}$ must also be "certain." Conclude that $f \vee g \sim f \vee g \vee f^{\prime}$, as required by GK. The next result makes precise this intuitive connection between GK, "impossibility" and "certainty," at least for MP preferences.

Theorem 2 Let $\succsim$ be a MP preference. Given any $f$ and $f^{\prime}$ in $L_{0}$, consider the following properties:

(i) $f \sim f \vee f^{\prime}$;

(ii) the event $\left\{s: f^{\prime}(s) \succ f(s)\right\}$ is null;

(iii) the event $\left\{s: f(s) \succsim f^{\prime}(s)\right\}$ is universal.

Then $($ iii $) \Longrightarrow(i) \Longrightarrow($ ii $)$; and the three properties are equivalent for any $f$ and $f^{\prime}$ in $L_{0}$ iff $\succsim$ satisfies $G K$.

Proof. (i) implies (ii): If $f \sim f \vee g$, then there is $P^{*} \in C$ such that

$$
\begin{aligned}
\min _{P \in C} \int u(f) d P & =\min _{P \in C} \int u\left(f \vee f^{\prime}\right) d P=\int u\left(f \vee f^{\prime}\right) d P^{*}=\int\left(u(f) \vee u\left(f^{\prime}\right)\right) d P^{*} \\
& \geq \int u(f) d P^{*} \geq \min _{P \in C} \int u(f) d P
\end{aligned}
$$

so that $\int u(f) d P^{*}=\int\left(u(f) \vee u\left(f^{\prime}\right)\right) d P^{*}$. Therefore, $P^{*}\left(\left\{s: f^{\prime}(s) \succ f(s)\right\}\right)=0$, which implies that $\left\{s: f^{\prime}(s) \succ f(s)\right\}$ is null.

(iii) implies (i): If $\left\{s: f(s) \succsim f^{\prime}(s)\right\}$ is universal, then $P(\{s: f(s) \succsim g(s)\})=1$ $\forall P \in C$, and $u\left(f^{\prime}(s)\right) \leq u(f(s)) P$-a.e. $\forall P \in C$. It follows that $f \sim f \vee g$. 
Let $\succsim$ satisfy GK. By Theorem 1, $\left\{s: f^{\prime}(s) \succ f(s)\right\}$ is null iff $\left\{s: f(s) \succsim f^{\prime}(s)\right\}$ is universal. Thus, given GK, (ii) implies (iii) and (i)-(iii) are equivalent. Conversely, assume (i)-(iii) are equivalent for any $f$ and $f^{\prime}$ in $L_{0}$. Then $f \sim f \vee f^{\prime} \Longrightarrow$ $P\left(\left\{s: f(s) \succsim f^{\prime}(s)\right\}\right)=1 \forall P \in C \Longrightarrow P\left(\left\{s:(f \vee g)(s) \succsim\left(f^{\prime} \vee g\right)(s)\right\}\right)=1 \forall P \in C$ $\Longrightarrow f \vee g \sim(f \vee g) \vee f^{\prime}$.

We conclude with a characterization of GK for all monotone (not necessarily MP) preferences. Say that the preference $\succsim$ on $L_{0}$ is monotone if $f \succsim g$ whenever $f(s) \succsim$ $g(s)$ for each $s \in S$.

Inspired by [5, p. 568], define a relation $\geqslant *$ on $L_{0}$ by

$$
f \geqslant * f^{\prime} \text { if } f \sim f \vee f^{\prime}
$$

For monotone preferences, GK is equivalent to transitivity of $\geqslant *$. In particular, under GK the symmetric part of $\geqslant *$ partitions $L_{0}$ into equivalence classes of acts that are indistinguishable according to $\succsim$.

Theorem 3 Let $\succsim$ be monotone. Then $\succsim$ satisfies $G K$ iff $\geqslant *$ is transitive.

Proof. Suppose $\geqslant^{*}$ is transitive. Then, by monotonicity, $f \vee g \geqslant * f$. Therefore, since $\geqslant *$ is transitive, $f \geqslant * g \Longrightarrow f \vee g \geqslant * g$, which proves GK.

Conversely, assume GK. ${ }^{2}$ Suppose $f \geqslant * g$ and $g \geqslant * h$, that is, $f \sim f \vee g$ and $g \sim g \vee h$. We have $f \sim f \vee g \succsim g \sim g \vee h$ by monotonicity. By GK, $g \sim g \vee h$ implies $g \vee f \sim g \vee h \vee f$, and, by monotonicity, $g \vee h \vee f \succeq h \vee f$. Therefore,

$$
f \sim f \vee g \sim g \vee h \vee f \succeq h \vee f \geqslant f
$$

which implies $f \sim h \vee f$, that is, $f \geqslant * h$.

\section{References}

[1] P. Artzner, F. Delbaen, J.M. Eber, D. Heath, and H. Ku, Coherent multiperiod risk adjusted values and Bellman's principle, Annals Oper. Res., forthcoming.

[2] L.G. Epstein and M. Schneider, Recursive multiple-priors, J. Econ. Theory 113 (2003), 1-31.

[3] P. Ghirardato and M. Marinacci, Risk, ambiguity, and the separation of utility and beliefs, Math. Oper. Research 26 (2001), 864-890.

\footnotetext{
${ }^{2}$ We use an argument of Kreps [5, p. 569].
} 
[4] I. Gilboa and D. Schmeidler, Maxmin expected utility with a non-unique prior, J. Math. Econ. 18 (1989), 141-153.

[5] D. Kreps, A representation theorem for 'preference for flexibility', Econometrica 47 (1979), 565-577.

[6] F. Maccheroni, M. Marinacci, and A. Rustichini, Ambiguity aversion, robustness, and the variational representation of preferences, Econometrica, forthcoming. 